\title{
A Cluster-based Multiuser Cooperative Network
}

\author{
Celal Eşli and Armin Wittneben \\ ETH Zurich, Communication Technology Laboratory, CH-8092 Zurich, Switzerland \\ Email: \{cesli,wittneben\}@nari.ee.ethz.ch
}

\begin{abstract}
A novel cluster-based coherent multi-user (MU) relaying system is proposed, where a number of source/destination pairs communicate concurrently over the same physical channel and a set of amplify-and-forward relays assist them. A secondary short-range wireless system with single/multi-hop unicast or broadcast ability is considered for the channel state information (CSI) dissemination. To reduce the CSI dissemination overhead, the relays are grouped in several independent clusters. Distributed diversity gain is attained at the destinations by applying phase rotations between clusters. The CSI dissemination is analyzed thoroughly, and compared with that of previous works on $\mathrm{MU}$ relaying. It is shown that for a given number of clusters and relays, the most uniform distribution of relays to clusters is optimum to minimize the CSI exchange traffic overhead and transmit energy consumption for CSI dissemination; besides it maximizes the average achievable rate for source/destination links. Furthermore, a new solution for a maxmin fairness based relay gain allocation is proposed and formulated as a semidefinite program.
\end{abstract}

\section{INTRODUCTION}

Cooperative relaying schemes have been proposed as a promising approach to supersede the conventional multipleinput multiple-output systems to achieve diversity and spatial multiplexing gains. In this work we focus on amplify-andforward (AF) relays assisted two-hop half-duplex transmission between source/destination (S/D) pairs. In the first phase the sources transmit data to the relays and in the second phase, the relays transmit the scaled and/or rotated data to the destinations. In order to achieve a spatial multiplexing gain, independent data streams are transmitted concurrently in the same frequency band. In [1], the conversion of spatial diversity to temporal diversity by relay phase rotations is proposed. A system with $N_{r}$ AF relays is shown to achieve the maximum distributed diversity gain of $N_{r}$ by using time-variant phaserotations at the relays [2].

A MU relaying scheme for the case of infinite number of relays is proposed in [3]. For finite number of singleantenna relays, a scheme which is based on zero-forcing (ZF) by performing a nullspace projection is presented in [4]. Multiple AF relays assist the communication between multiple single-antenna S/D pairs. Choosing the relay gain factors accordingly, the S/D links are orthogonalized which removes the interference between different S/D pairs. A minimum number of relays is needed to achieve this. When there are more relays, the gain factors can be further optimized [5].

The aforementioned multi-user relaying systems require the so called global channel state information (GCSI) at each relay to determine the relay gain factors, which means that each relay knows not only its local CSI (LCSI), but also
LCSIs of all other relays in the system. The dissemination of these LCSIs can be performed by a secondary short-range low-power wireless technology. Depending on the system features, the LCSIs can be disseminated by unicast or broadcast communication between relays. It has been shown in [6] that unicast communications can be preferable for certain conditions. On the other hand, considering a dense network, the number of required LCSI exchanges (and accordingly the energy consumption) increases polynomially with the number of nodes in the system. Moreover, the topological conditions may prevent to exchange LCSI in such a dense network.

In this paper, we propose a novel cluster based multi-user relaying (C-MUR) system, where the relays are grouped in independent clusters. Applying phase rotations between clusters, distributed diversity gain is attained at the destinations. As opposed to the previous works on multi-user ZF relaying (MUZFR), a new solution for relay gain allocation is proposed which does not require nullspace projection. Considering the fairness between different links, a maxmin link-rate optimization problem is formulated as a semidefinite program (SDP). Furthermore, a LCSI dissemination analysis in terms of traffic overhead and transmit energy consumption is performed and compared to that of MUZFR with GCSI. It is shown that for a given number of clusters and relays, the most uniform distribution of relays to clusters is optimum to minimize the LCSI exchange overhead and energy consumption. Moreover it maximizes the average link rates.

Notation: The superscripts $(\cdot)^{*},(\cdot)^{T}$ and $(\cdot)^{H}$ stand for conjugate complex, matrix transpose and conjugate complex transpose, respectively. The operators $\odot, \mathrm{E}_{\{x\}}[\cdot], \operatorname{diag}\{\mathbf{x}\}, \operatorname{tr}\{\mathbf{X}\}$ denote the elementwise product, expectation with respect to $x$, a diagonal matrix with $\mathbf{x}$ on its diagonal, and trace of $\mathbf{X}$, respectively. $x \sim \mathcal{C N}\left(0, \sigma^{2}\right)$ stands for a zero-mean complex normal circularly symmetric distribution with variance $\sigma^{2}$. The operation $\bmod (a, b)$ stands for the modulo operation that finds the remainder of division of $a$ by $b$.

\section{SYSTEM MODEL}

We consider a wireless network with $2 N+N_{r}$ singleantenna nodes, where $N$ S/D pairs communicate concurrently on the same physical channel, and $N_{r}$ amplify-and-forward relay nodes assist the communication in a half-duplex scheme. The relays are grouped together in $C$ clusters each with $n_{k}$ relays such that $\sum_{k=1}^{C} n_{k}=N_{r}$. Each cluster is assumed to be unaware and independent of the others. It is also assumed that there are no direct links between sources and destinations due to shadowing effects or topological conditions. Fig. 1 summarizes the network configuration. 


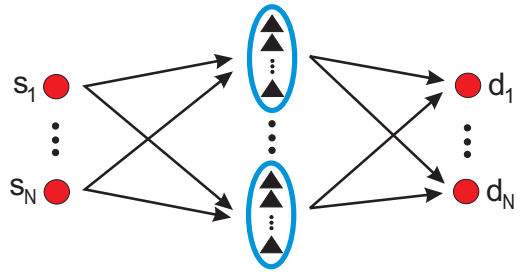

Fig. 1. Cluster based two-hop network configuration with half-duplex relays.

The communication follows a two-hop relay traffic pattern. In the first hop, each source transmits $T$ symbols consecutively. The $n_{k} \times T$ dimensional received signal block at the $k$ th cluster over $T$ time slots is $\mathbf{R}_{k}=\mathbf{H}_{\mathrm{sr}}^{(k)} \mathbf{S}+\mathbf{W}_{k}$, where $\mathbf{R}_{k}=\left[\mathbf{r}_{k}[1] \cdots \mathbf{r}_{k}[T]\right], \mathbf{r}_{k}[i]=\left[r_{k, 1}[i] \cdots r_{k, n_{k}}[i]\right]^{T}, r_{k, j}[i]$ is the $i$ th received symbol of the $j$ th relay in the $k$ th cluster; $\mathbf{S}=[\mathbf{s}[1] \cdots \mathbf{s}[T]], \mathbf{s}[i]=\left[s_{1}[i] \cdots s_{N}[i]\right]^{T}, s_{m}[i]$ is the $i$ th symbol of the $m$ th source; $\mathbf{H}_{\mathrm{sr}}^{(k)} \in \mathbb{C}^{n_{k} \times N}$ is the uplink channel matrix from the sources to the $k$ th cluster, and $\mathbf{W}_{k}$ is the correspoding relay noise matrix with i.i.d. $\mathcal{C N}\left(0, \sigma_{w}^{2}\right)$ entries.

Before the relays transmit their received symbols through the downlink channel $\mathbf{H}_{\mathrm{rd}}^{(k)} \in \mathbb{C}^{N \times n_{k}}$ to the destinations, the signals are multiplied with the gain factors $g_{k, j}$ s to obtain $\mathbf{Y}_{k}=\mathbf{G}_{k} \mathbf{R}_{k}$, where $\mathbf{G}_{k}=\operatorname{diag}\left\{\left[g_{k, 1} \cdots g_{k, n_{k}}\right]\right\}$. The calculation of the gain factors is explained in Section III. They depend on the uplink and downlink channel matrices. The channel matrices $\mathbf{H}_{\mathrm{sr}}^{(k)}$ and $\mathbf{H}_{\mathrm{rd}}^{(k)}$ have i.i.d. $\mathcal{C N}(0,1)$ entries and stay constant during at least $2 T$ symbol transmission cycles, where we assume block fading channels. Moreover, we assume that each relay knows the CSI of its uplink/downlink channels as well as the other relays' that belong to its own cluster, i.e., $\mathbf{H}_{\mathrm{sr}}^{(k)}, \mathbf{H}_{\mathrm{rd}}^{(k)}$ are known to all relays in the $k$ th cluster. We call this local cluster CSI knowledge. Note that since the channel coefficients are constant over $2 T$ symbol times, the calculated $\mathbf{G}_{k}$ stays the same during this period.

If $\mathbf{Y}_{k}$ is transmitted to the destinations without any further signal processing, the compound channel coefficients from all clusters would add up to a single coefficient which stays constant during $T$ symbol periods. This prevents any temporal diversity. In [1], time variant phase offsets are introduced at the relays to create an artificial time-variant channel, which is then utilized to achieve diversity by an outer code. Here, we use this concept not between the relays but between the clusters. The spatial diversity is transformed to temporal diversity by time variant and cluster specific phase variations, i.e., the same phase variation is used for all relays in the same cluster. Thus, before transmission, $\mathbf{Y}_{k}$ is multiplied with time/cluster specific $p_{k, i}$ s such that $\mathbf{P}_{k} \odot \mathbf{Y}_{k}$ is transmitted to the destinations, where $\mathbf{P}_{k}=\left[\left[p_{k, 1} \ldots p_{k, T}\right]^{T} \ldots\left[p_{k, 1} \ldots p_{k, T}\right]^{T}\right]^{T} \in \mathbb{C}^{n_{k} \times T}$.

The sum transmit power per symbol of all relays in the $k$ th cluster is equal to $P_{k}$. It is assumed that each cluster transmits with the same power $P_{k}=\sigma_{s}^{2} N / C$, where $\sigma_{s}^{2}$ is the average power of the source transmit symbols. We use this constraint to keep the total power consumption of the network constant when adding relays to clusters.

The destinations receive a superposition of all signals from
$C$ clusters. Hence, the received signal block over $T$ time slots is represented as

$\mathbf{D}=\sum_{k=1}^{C}\left(\mathbf{H}_{\mathrm{rd}}^{(k)}\left(\mathbf{P}_{k} \odot\left(\mathbf{G}_{k} \mathbf{H}_{\mathrm{sr}}^{(k)} \mathbf{S}\right)\right)+\mathbf{H}_{\mathrm{rd}}^{(k)}\left(\mathbf{P}_{k} \odot\left(\mathbf{G}_{k} \mathbf{W}_{k}\right)\right)\right)+\mathbf{N}$,

where $\mathbf{D}=[\mathbf{d}[1] \cdots \mathbf{d}[T]], \mathbf{d}[i]=\left[d_{1}[i] \cdots d_{N}[i]\right]^{T}, d_{m}[i]$ is the $i$ th received symbol of the $m$ th destination, and $\mathbf{N}$ is the corresponding noise matrix at destinations with i.i.d. $\mathcal{C N}\left(0, \sigma_{n}^{2}\right)$ entries. In the sequel, it is assumed that $\sigma_{w}^{2}=\sigma_{n}^{2}$. The sources do not have CSI knowledge, whereas the destinations know the compound CSI, i.e., $\mathbf{H}_{\mathrm{rd}}^{(k)} \mathbf{G}_{k} \mathbf{H}_{\mathrm{sr}}^{(k)} \forall k$. There is no symbol exchange between the relays or destinations.

In order to achieve the maximum available diversity we choose $T=C$. A simple choice for the compound phase rotation matrix $\mathbf{P}=\left[\left[p_{1,1} \cdots p_{1, C}\right]^{T} \cdots\left[p_{C, 1} \cdots p_{C, C}\right]^{T}\right] \in \mathbb{C}^{C \times C}$ is an identity matrix [1]. Choosing $\mathbf{P}$ as an identity matrix, the columns of $\mathbf{D}$ simplify to

$\mathbf{d}[i]=\mathbf{H}_{\mathrm{rd}}^{(i)} \mathbf{G}_{i} \mathbf{H}_{\mathrm{sr}}^{(i)} \mathbf{s}[i]+\mathbf{H}_{\mathrm{rd}}^{(i)} \mathbf{G}_{i} \mathbf{w}_{i}[i]+\mathbf{n}[i]$ for $i=1, \ldots, C$.

With the chosen $\mathbf{P}$ each time slot is assigned to a single cluster. Thus, the total transmit power of this single cluster becomes $P_{k}=\sigma_{s}^{2} N$. Since the $i$ th symbol interval is assigned to the $k$ th cluster, in the sequel we use the indices $i$ and $k$ interchangeably. Finally, transmitting over $C$ time slots i.i.d. symbols $s_{m}[i] \sim \mathcal{C N}\left(0, \sigma_{s}^{2}\right)$, the mutual information between the $m$ th S/D pair for this artificial time-selective channel is $I_{m}=\frac{1}{2 C} \sum_{i=1}^{C} \log _{2}\left(1+\mathrm{SNR}_{m}^{(i)}\right)$, where $\mathrm{SNR}_{m}^{(i)}$ is the signalto-noise ratio of the $m$ th link induced by the $i$ th cluster in the $i$ th symbol interval.

\section{Relay Gain Allocation Per Cluster}

In the proposed cluster-based MU relaying system, each cluster is independent and unaware of the others. Thus, each cluster per se tries to optimize its gain vector such that the minimum link rate is maximized from its perspective.

Let $\mathbf{h}_{\mathrm{sr}}^{k, l}$ and $\mathbf{h}_{\mathrm{rd}}^{k, l}$ be the $l$ th column vector of $\mathbf{H}_{\mathrm{sr}}^{(k)}$ and the transpose of the lth row vector of $\mathbf{H}_{\mathrm{rd}}^{(k)}$, respectively. Considering the $k$ th cluster, the instantaneous rate of the $m$ th S/D link is $\mathrm{R}_{m}^{(k)}=\frac{1}{2} \log _{2}\left(1+\operatorname{SINR}_{m}^{(k)}\right)$, and the instantenous signalto-interference plus noise ratio $\operatorname{SINR}_{m}^{(k)}$ from the perspective of the $k$ th cluster is given by

$$
\operatorname{SINR}_{m}^{(k)}=\frac{\sigma_{s}^{2} \mathbf{g}_{k}^{H} \mathbf{H}_{m, m}^{(k)} \mathbf{g}_{k}}{\sigma_{s}^{2} \sum_{n \neq m} \mathbf{g}_{k}^{H} \mathbf{H}_{m, n}^{(k)} \mathbf{g}_{k}+\sigma_{n}^{2}\left(1+\mathbf{g}_{k}^{H} \mathbf{M}_{m}^{(k)} \mathbf{g}_{k}\right)},
$$

where $\mathbf{H}_{m, n}^{(k)}=\left(\mathbf{h}_{\mathrm{rd}}^{k, m} \odot \mathbf{h}_{\mathrm{sr}}^{k, n}\right)^{*}\left(\mathbf{h}_{\mathrm{rd}}^{k, m} \odot \mathbf{h}_{\mathrm{sr}}^{k, n}\right)^{T}, \mathbf{g}_{k}=\left[g_{k, 1} \cdots g_{k, n_{k}}\right]^{T}$, and $\mathbf{M}_{m}^{(k)}=\operatorname{diag}\left\{\left|\mathbf{h}_{\mathrm{rd}}^{\mathrm{k}, \mathrm{m}}\right|^{2}\right\}$. Each cluster independently solves the following optimization problem

$$
\max _{\mathbf{g}_{k}} \min _{1 \leq i \leq N} \mathbf{R}_{m}^{(k)} \quad \text { subject to } \mathbf{g}_{k}^{H} \mathbf{Q}_{k} \mathbf{g}_{k} \leq P_{k},
$$

where $\mathbf{Q}_{k}=\sigma_{s}^{2}\left(\left(\mathbf{H}_{\mathrm{sr}}^{(k)}\right)^{H} \mathbf{H}_{\mathrm{sr}}^{(k)}\right) \odot \mathbf{I}_{n_{k}}+\sigma_{n}^{2} \mathbf{I}_{n_{k}}$. The problem (1) is monotonically equivalent to

$$
\max _{\mathbf{g}_{k}} \min _{1 \leq i \leq N} \mathrm{SNR}_{m}^{(k)} \quad \text { subject to } \mathbf{g}_{k}^{H} \mathbf{Q}_{k} \mathbf{g}_{k} \leq P_{k} .
$$


Using the relation $\mathbf{g}_{k}^{H} \mathbf{H}_{m, n}^{(k)} \mathbf{g}_{k}=\operatorname{tr}\left(\mathbf{H}_{\mathrm{m}, \mathrm{n}}^{(\mathrm{k})} \mathbf{g}_{\mathrm{k}} \mathbf{g}_{\mathrm{k}}^{\mathrm{H}}\right)=\operatorname{tr}\left(\mathbf{H}_{\mathrm{m}, \mathrm{n}}^{(\mathrm{k})} \mathbf{G}_{\mathrm{k}}\right)$ and introducing a dummy variable $\tau,(2)$ is reformulated as

$$
\begin{aligned}
& \max _{\tau, \mathbf{g}_{k}} \tau \quad \text { subject to } \quad \operatorname{tr}\left(\tilde{\mathbf{Q}}_{k} \mathbf{G}_{k}\right) \leq P_{k} \\
& \frac{\sigma_{s}^{2}}{\tau} \operatorname{tr}\left(\mathbf{H}_{m, m}^{(k)} \mathbf{G}_{k}\right)-\sigma_{s}^{2} \sum_{n \neq m} \operatorname{tr}\left(\mathbf{H}_{m, n}^{(k)} \mathbf{G}_{k}\right)-\sigma_{n}^{2} \operatorname{tr}\left(\mathbf{M}_{m}^{(k)} \mathbf{G}_{k}\right) \geq \sigma_{n}^{2} \forall m \\
& \operatorname{rank}(\mathbf{G})=1, \quad \mathbf{G}_{k}=\mathbf{G}_{k}^{*}, \quad \mathbf{G}_{k} \succeq 0,
\end{aligned}
$$

which is a nonconvex problem because of the rank constraint. Omitting the rank constraint (Lagrangian relaxation), the problem (3) can be converted to a quasi-convex SDP problem. This relaxed problem is equivalent to a SDP feasibility problem when $\tau$ is given a priori and can be efficiently solved by SeDuMi [7]. Thus, we use the following bisection algorithm to solve the Lagrangian relaxed problem:

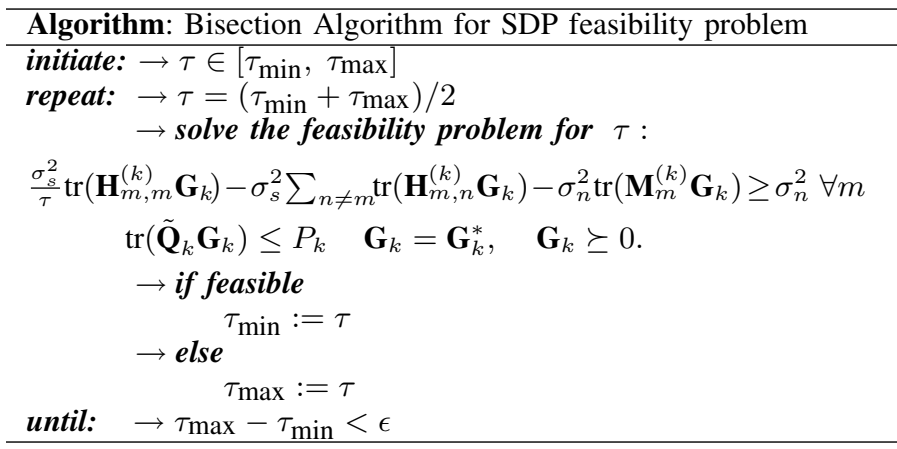

In general, $\tau_{\min }$ is set to 0 and $\tau_{\max }$ is chosen large enough according to the operation SNR value. Since we drop the rank constraint from the original problem (3), the solution of the relaxed problem may or may not result in a rank$1 \mathbf{G}_{k}^{\text {opt }}$ matrix. To choose the $\mathbf{g}_{k}^{\text {opt }}$ out of $\mathbf{G}_{k}^{\text {opt }}$ we use the suboptimal randomization technique proposed in [8]. The eigenvalue decomposition of $\mathbf{G}_{k}^{\mathrm{opt}}=\mathbf{U} \boldsymbol{\Gamma} \mathbf{U}^{H}$ is computed, and $\mathbf{g}_{k}^{\text {opt }}$ is chosen such that $\mathbf{g}_{k}^{\text {opt }}=\mathbf{U} \Gamma^{\frac{1}{2}} \mathbf{w}_{k}$, where $\mathbf{w}_{k}[t]=e^{j \theta_{k, t}}$ for independently and uniformly distributed phases $\theta_{k, t}$ on $[0,2 \pi)$. Independent from any realization of $\mathbf{w}_{k}$, it is assured that $\left(\mathbf{g}_{k}^{\text {opt }}\right)^{H} \mathbf{g}_{k}^{\text {opt }}=\operatorname{tr}\left(\mathbf{G}_{k}^{\text {opt }}\right)$. After generating $\mathbf{g}_{k}^{\text {opt }}$, any violation of constraints should be checked and $\mathbf{g}_{k}^{\text {opt }}$ can be scaled to satisfy the most violated constraint accordingly.

\section{The CSI Dissemination BetweEn Relays}

We consider a network with $C$ relay clusters each with $n_{i}$, $i=1, \ldots, C$, relays. It is assumed that the relays perfectly estimate their backward and forward channels. With the independency of clusters, each relay in the $i$ th cluster exchanges LCSI only with the other $n_{i}-1$ relays in the same cluster.

In a single-hop unicast scheme, each node needs to establish point-to-point links with all the other nodes, which results in a high exchange overhead and transmit energy consumption. Instead, the relays can also establish multiple short-distance hops to disseminate their LCSI (multi-hop unicast). In other words, the relays help each other by forwarding the received LCSI of its neighbours to others. Moreover, if the relays are able to broadcast data, they can establish point-to-multipoint links, which in turn reduces the amount of exchange load w.r.t.

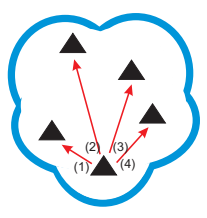

Single-hop Unicast
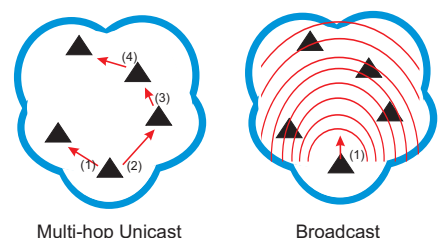

Fig. 2. The schemes to disseminate LSCI between relays: For the current scenario, unicast schemes use 4 channel use, whereas broadcast uses only one.

unicast schemes. See Fig. 2 for the interpretation of different dissemination schemes.

\section{A. The CSI Exchange Load}

1) Unicast: Assuming a secondary short-range wireless system with only unicast ability (single or multi-hop), the measure of local CSI exchange load (CSIEL) of the $i$ th cluster is defined as $\phi\left(n_{i}\right)=2 \alpha N n_{i}\left(n_{i}-1\right)$, where the factor 2 stands for the need for exchanging both uplink and downlik LCSI. $N$ is the number of links, and $\alpha$ is a constant representing the number of units which can be exchanged in each attempt. For simplicity, we assume that exchanges are error-free.

Proposition 1: Given $N_{r}$ and $C$, the most uniform distribution of relays to clusters is the optimum cluster-relay configuration (OCC) that minimizes the CSIEL independently of $N$ and $\alpha$. Thus, if $\bmod \left(N_{r}, C\right)=0$, we have $C$ clusters each with $N_{r} / C$ relays; otherwise there are $\bmod \left(N_{r}, C\right)$ clusters each with $\left\lceil N_{r} / C\right\rceil$ relays, and $C-\bmod \left(N_{r}, C\right)$ clusters each with $\left\lfloor N_{r} / C\right\rfloor$ relays.

Proof: Assume that for given $C$ and $N_{r}$, there exists a cluster relay configuration $\tilde{\mathcal{C}}=\left\{m_{1}, \ldots, m_{C}\right\}$ with the total exchange load $\sum_{i=1}^{C} \phi\left(m_{i}\right)$ which is smaller than or equal to $\sum_{i=1}^{C} \phi\left(n_{i}\right)$ obtained by the proposed OCC $\mathcal{C}=\left\{n_{1}, \ldots, n_{C}\right\}$. Let $m_{i}=n_{i}+r_{i}$ and $\phi\left(m_{i}\right)=\phi\left(n_{i}\right)+\beta_{i}$, where $\beta_{i}$ is bounded as

$$
\beta_{i} \geq r_{i} \gamma_{1}\left(n_{i}\right), \quad r_{i} \geq 0 \quad \text { or } \quad \beta_{i} \geq r_{i} \gamma_{2}\left(n_{i}\right), \quad r_{i}<0,
$$

where $r_{i} \in \mathbb{Z} . \gamma_{1}\left(n_{i}\right)=4 \alpha N n_{i}$ and $\gamma_{2}\left(n_{i}\right)=4 \alpha N\left(n_{i}-1\right)$ are functions of $n_{i}$ and equal to the absolute CSIEL difference obtained by incrementing or decrementing $n_{i}$ by 1 , respectively. Henceforth, we drop the $\left(n_{i}\right)$ designation of $\gamma_{1}\left(n_{i}\right)$ and $\gamma_{2}\left(n_{i}\right)$ for notational simplicity. Rearranging the total load of $\tilde{\mathcal{C}}$ as $\sum_{i=1}^{C} \phi\left(m_{i}\right)=\sum_{i \in \mathcal{P}} \phi\left(m_{i}\right)+\sum_{i \in \mathcal{P}^{\prime}} \phi\left(m_{i}\right)$, we obtain

$$
\begin{aligned}
\sum_{i=1}^{C} \phi\left(m_{i}\right) & =\sum_{i \in \mathcal{P}}\left(\phi\left(n_{i}\right)+r_{i} \gamma_{1}+\epsilon_{i}\right)+\sum_{i \in \mathcal{P}^{\prime}}\left(\phi\left(n_{i}\right)+r_{i} \gamma_{2}+\epsilon_{i}\right) \\
& =\sum_{i \in \mathcal{P}} r_{i} \gamma_{1}+\sum_{i \in \mathcal{P}^{\prime}} r_{i} \gamma_{2}+\sum_{i=1}^{C} \phi\left(n_{i}\right)+\sum_{i=1}^{C} \epsilon_{i}
\end{aligned}
$$

where $\epsilon_{i} \in \mathbb{R}^{+} \forall i, \mathcal{P}$ is the index set of clusters with positive $r_{i} \mathrm{~s}$, and $\mathcal{P}^{\prime}$ is the index set of remainder clusters. Focusing on the first two summands of (4),

$$
\begin{aligned}
& \sum_{i \in \mathcal{P}} r_{i} \gamma_{1}+\sum_{i \in \mathcal{P}^{\prime}} r_{i} \gamma_{2}=\sum_{i \in \mathcal{P} \cap \mathcal{N}} r_{i} \gamma_{1}+\sum_{i \in \mathcal{P} \cap \mathcal{N}^{\prime}} r_{i} \gamma_{1}+\sum_{i \in \mathcal{P}^{\prime} \cap \mathcal{N}} r_{i} \gamma_{2}+\sum_{i \in \mathcal{P}^{\prime} \cap \mathcal{N}^{\prime}} r_{i} \gamma_{2} \\
& =4 \alpha N\left(\sum_{i \in \mathcal{P}} r_{i}\left\lceil N_{r} / C\right\rceil-\sum_{i \in \mathcal{P} \cap \mathcal{N}^{\prime}} r_{i}+\sum_{i \in \mathcal{P}^{\prime}} r_{i}\left(\left\lceil N_{r} / C\right\rceil-1\right)-\sum_{i \in \mathcal{P}^{\prime} \cap \mathcal{N}^{\prime}} r_{i}\right)
\end{aligned}
$$


where $\mathcal{N}$ and $\mathcal{N}^{\prime}$ are the index sets of clusters according to the OCC with $\left\lceil N_{r} / C\right\rceil$ and $\left\lfloor N_{r} / C\right\rfloor=\left\lceil N_{r} / C\right\rceil-1$ relays, respectively. Since $\sum_{i=1}^{C} m_{i}=\sum_{i=1}^{C} n_{i}=N_{r}$, the condition $\sum_{i \in \mathcal{P}}\left|r_{i}\right|=\sum_{i \in \mathcal{P}^{\prime}}\left|r_{i}\right|$ should also be satisfied. Introducing this fact, we obtain

$$
\begin{aligned}
\sum_{i \in \mathcal{P}} r_{i} \gamma_{1}+\sum_{i \in \mathcal{P}^{\prime}} r_{i} \gamma_{2} & =4 \alpha N\left(\sum_{i \in \mathcal{P}}\left|r_{i}\right|-\sum_{i \in \mathcal{P} \cap \mathcal{N}^{\prime}}\left|r_{i}\right|+\sum_{i \in \mathcal{P}^{\prime} \cap \mathcal{N}^{\prime}}\left|r_{i}\right|\right) \\
& =4 \alpha N\left(\sum_{i \in \mathcal{P} \cap \mathcal{N}}\left|r_{i}\right|+\sum_{i \in \mathcal{P}^{\prime} \cap \mathcal{N}^{\prime}}\left|r_{i}\right|\right)>0
\end{aligned}
$$

Combining (5) with (4), we have $\sum_{i=1}^{C} \phi\left(m_{i}\right)>\sum_{i=1}^{C} \phi\left(n_{i}\right)$, which contradicts with our assumption that there exists a $\tilde{\mathcal{C}}$ which has a smaller or equal CSI exchange load than $\mathcal{C}$.

Proposition 2: Given $N_{r}$ and the design constraint that relays are distributed to clusters as uniform as possible, a network with $C>0$ clusters has more CSIEL than any network with $C+\beta$ clusters, where $\beta \in \mathbb{Z}^{+}$.

Proof (Sketch): The proof follows from the definiton of $\phi(n)$. The proposition claims that

$$
\sum_{i \in \mathcal{R}}^{C} \phi\left(m_{i}\right)+\sum_{i \in \mathcal{R}^{\prime}}^{C} \phi\left(m_{i}\right)>\sum_{i \in \mathcal{Q}}^{C+\beta} \phi\left(n_{i}\right)+\sum_{i \in \mathcal{Q}^{\prime}}^{C+\beta} \phi\left(n_{i}\right)
$$

where $m_{i} \mathrm{~s}$ and $n_{i} \mathrm{~s}$ are chosen according to OCC stated in Proposition 1, and $\mathcal{R}, \mathcal{R}^{\prime}, \mathcal{Q}, \mathcal{Q}^{\prime}$ stand for the index set of clusters with $\eta_{1}=\left\lceil N_{r} / C\right\rceil, \eta_{2}=\left\lfloor N_{r} / C\right\rfloor, \eta_{3}=\left\lceil N_{r} /(C+\beta)\right\rceil$, and $\eta_{4}=\left\lfloor N_{r} /(C+\beta)\right\rfloor$ relays, respectively. Defining $\xi_{1}=$ $\bmod \left(N_{r}, C\right), \xi_{2}=C-\bmod \left(N_{r}, C\right), \xi_{3}=\bmod \left(N_{r}, C+\beta\right)$, $\xi_{4}=C+\beta-\bmod \left(N_{r}, C+\beta\right)$, the inequality boils down to

$$
\eta_{2}\left(N_{r}-\xi_{2}\right)>\eta_{4}\left(N_{r}-\xi_{4}\right)
$$

which can be further modified to the following expression after some algebraic manipulations and setting $\beta=1$,

$$
\begin{aligned}
\left(\left\lfloor\frac{N_{r}}{C}\right\rfloor\right. & \left.-\left\lfloor\frac{N_{r}}{C+1}\right\rfloor\right)\left(2 N_{r}-C\right)>C\left(\left\lfloor\frac{N_{r}}{C}\right\rfloor-\left\lfloor\frac{N_{r}}{C+1}\right\rfloor\right) \\
& \times\left(\left\lfloor\frac{N_{r}}{C}\right\rfloor+\left\lfloor\frac{N_{r}}{C+1}\right\rfloor\right)-\left\lfloor\frac{N_{r}}{C+1}\right\rfloor\left(\left\lfloor\frac{N_{r}}{C+1}\right\rfloor+1\right) .
\end{aligned}
$$

For any $C>N_{r} / 2$, (7) holds true. Moreover, for any $C \leq N_{r} / 2$ that leads $\lfloor N / C\rfloor=\lfloor N /(C+1)\rfloor$, (7) can be shown to be true. In order to prove the proposition for any $C \leq N_{r} / 2$ which results in $\lfloor N / C\rfloor \neq\lfloor N /(C+1)\rfloor$, it is sufficient to show that

$$
2 N_{r} / C-1 \geq\left\lfloor N_{r} / C\right\rfloor+\left\lfloor N_{r} /(C+1)\right\rfloor .
$$

Redefining $\left\lfloor N_{r} /(C)\right\rfloor$ and $\left\lfloor N_{r} /(C+1)\right\rfloor$ as $N_{r} /(C)-a$ and $N_{r} /(C+1)-b, 0 \leq a<1,0 \leq b<1$, respectively, (8) becomes $N_{r} / C-N_{r} /(C+1) \geq 1-a-b$. This can be shown to hold true immediately, which proves the inequality (6) for $\beta=1$. The proof is concluded by induction for $\beta>1$.

With Propositions 1-2, the minimum CSIEL is attained by the most uniform distribution of relays to $C_{\max }$ clusters, where $C_{\max }=\left\lfloor N_{r} / N_{\min }\right\rfloor$ and $N_{\min }$ is the minimum allowable cluster size determined by the network designer.

2) Broadcast: Assuming the same conditions as in the unicast case, the measure of CSIEL of the $i$ th cluster with broadcast dissemination, can be defined as $\psi\left(n_{i}\right)=2 \alpha N n_{i}$. $\psi\left(n_{i}\right)$ changes linearly with $n_{i}$, and the total network CSIEL is not affected by the size of different clusters.

\section{B. Energy Consumption of LCSI Dissemination}

Let $E_{c}$ be the so called sensitivity level of any node, which is the minimum amount of receive energy that the node needs to decode the received symbols correctly. The transmit energy reduces proportionally with $d^{-v}$, where $d$ is the distance between the transmitter and receiver, and $v \geq 2$ is the path loss exponent. Thus, the energy consumption per one unit of exchange is $\alpha E_{c} d^{v}$.

1) Single-hop Unicast: Considering the $i$ th cluster with $n_{i}$ relays, which are arbitrarily located and use single-hop unicast communication inbetween, the total energy consumption of the cluster for CSI dissemination is $E_{\mathrm{csi}}^{s u}\left(n_{i}\right)=$ $2 \alpha N E_{c} \sum_{i=1}^{n_{i}} \sum_{j=1, j \neq i}^{n_{i}} d_{i, j}^{v}$ where $d_{i, j}$ is the distance between the $i$ th and the $j$ th relay.

2) Multi-hop Unicast: Dissemination with multiple hops and establishing short distance point-to-point links reduce the transmit energy consumption drastically w.r.t. to single-hop unicast. Defining $\mathcal{M}_{i}$ as the lowest-energy-optimal set of distances of the hops that the $i$ th relay needs to disseminate its LCSI, the general energy consumption expression for multihop unicast scheme is $E_{\mathrm{csi}}^{m u}\left(n_{i}\right)=2 \alpha N E_{c} \sum_{i=1}^{n_{i}} \sum_{j \in \mathcal{M}_{i}} d_{j}^{v}$. Since the derivation of this optimal set $\mathcal{M}_{i}$ is out of the scope of this paper, we assume that it is readily available and known.

3) Broadcast: The total energy consumption of a $n_{i}$ relay cluster using broadcast communication for CSI dissemination is $E_{\mathrm{csi}}^{b}\left(n_{i}\right)=2 \alpha N E_{c} \sum_{i=1}^{n_{i}} d_{i, \max }^{v}$, where $d_{i, \max }=\max \left(d_{i, j}\right), \forall j \neq i, j=1, \ldots, n_{i}$.

To simplify the analysis, we further consider the following scenario for energy consumption calculations. Assume that all relays are located on a straight line with equal distances $r$ between the neighbouring relays as shown in Fig. 1. Then $E_{\mathrm{csi}}^{s u}\left(n_{i}\right), E_{\mathrm{csi}}^{m u}\left(n_{i}\right)$, and $E_{\mathrm{csi}}^{b}\left(n_{i}\right)$ can be re-defined as

$$
\begin{gathered}
\tilde{E}_{\mathrm{csi}}^{s u}\left(n_{i}\right)=4 \alpha N E_{c} r^{v}\left(\sum_{k=1}^{\left\lceil n_{i} / 2\right\rceil-\bmod \left(n_{i}, 2\right)}\left(\sum_{i=1}^{n_{i}-k}\left(i^{v}\right)+\sum_{i=1}^{k-1}\left(i^{v}\right)\right)\right. \\
\left.+\bmod \left(n_{i}, 2\right) \sum_{i=1}^{\left\lfloor n_{i} / 2\right\rfloor}\left(i^{v}\right)\right), \\
\tilde{E}_{\mathrm{csi}}^{m u}\left(n_{i}\right)=2 \alpha N E_{c} n_{i}\left(n_{i}-1\right) r^{v}, \\
\tilde{E}_{\mathrm{csi}}^{b}\left(n_{i}\right)=2 \alpha N E_{c} r^{v}\left(2 \sum_{i=\left\lceil n_{i} / 2\right\rceil}^{n_{i}-1}\left(i^{v}\right)+\bmod \left(n_{i}, 2\right)\left(\left\lfloor n_{i} / 2\right\rfloor\right)^{v}\right),
\end{gathered}
$$

respectively. This scenario will be used for the corresponding simulations in Section V.

Proposition 3: Given $N_{r}, C$, and $r$, the OCC minimizes all $\tilde{E}_{\mathrm{csi}}^{s u}(n), \tilde{E}_{\mathrm{csi}}^{m u}(n)$, and $\tilde{E}_{\mathrm{csi}}^{b}(n)$ independently of $v, E_{c}, N, \alpha$.

Proof (Sketch): It can be shown that for all $\tilde{E}_{\mathrm{csi}}^{s u}(n), \quad \tilde{E}_{\mathrm{csi}}^{m u}(n), \quad$ and $\tilde{E}_{\mathrm{csi}}^{b}(n)$ any $\left\{n_{1}, n_{2}, n_{3} \in\right.$ $\left.\mathbb{N} \mid n_{3}>n_{2}>n_{1}, n_{3}-n_{2}=n_{2}-n_{1}=1, n_{1}, n_{2}, n_{3}\right\}$ results in

$$
\begin{aligned}
\tilde{E}_{\mathrm{csi}}^{s u}\left(n_{3}\right)-\tilde{E}_{\mathrm{csi}}^{s u}\left(n_{2}\right) & >\tilde{E}_{\mathrm{csi}}^{s u}\left(n_{2}\right)-\tilde{E}_{\mathrm{csi}}^{s u}\left(n_{1}\right), \\
\tilde{E}_{\mathrm{csi}}^{m u}\left(n_{3}\right)-\tilde{E}_{\mathrm{csi}}^{m u}\left(n_{2}\right) & >\tilde{E}_{\mathrm{csi}}^{m u}\left(n_{2}\right)-\tilde{E}_{\mathrm{csi}}^{m u}\left(n_{1}\right), \\
\tilde{E}_{\mathrm{csi}}^{b}\left(n_{3}\right)-\tilde{E}_{\mathrm{csi}}^{b}\left(n_{2}\right) & >\tilde{E}_{\mathrm{csi}}^{b}\left(n_{2}\right)-\tilde{E}_{\mathrm{csi}}^{b}\left(n_{1}\right) .
\end{aligned}
$$




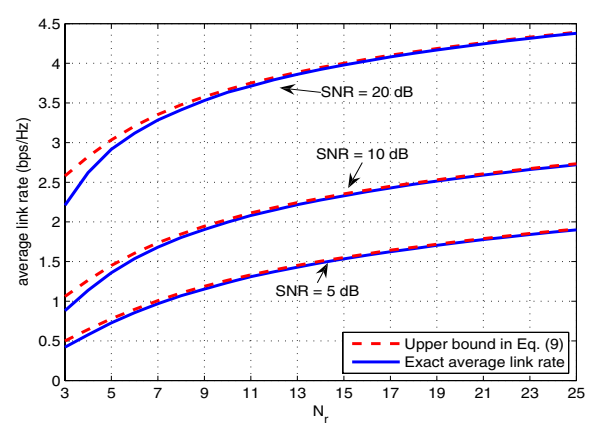

Fig. 3. Comparison of the upper bound and exact link rate with increasing $N_{r}(N=2, C=1$.$) .$

The proofs directly follow from the definition of the total energy consumptions and their polynomial behaviour. Next, the proposition can be proved by contradiction with similar steps to Proposition 1.

A similar statement to Proposition 2 can also be applied to the transmit energy consumption.

\section{Performance of optimum cluster configuration}

The proposed cluster based MU system unavoidably suffers from an array gain loss, when compared with MU relaying with GCSI. This is due to the fact that the coherent combination of all relays in the system has been disturbed and reduced to coherent combination of the relays in a single cluster. Thus, the less clusters there are in the system, the higher is the array gain and so is the average rate correspondingly. Besides, the interesting point here is the impact of the OCC in Proposition 1 on the average rate performance of the links. In the following, we show that the OCC also maximizes the average link rates, given that all relays are at the same distance to all sources, the $P_{k}$ s are equal/fixed and $N, \sigma_{s}^{2} / \sigma_{n}^{2}$ are fixed.

Using Jensen's inequality, the channel averaged mutual information of the $m$ th link is bounded by

$$
\mathrm{E}_{\mathbf{H}}\left\{I_{m}\right\} \leq \frac{1}{2 C} \sum_{i=1}^{C} \log _{2}\left(1+\mathrm{E}_{\mathbf{H}}\left\{\operatorname{SNR}_{m}^{(i)}(\mathbf{H})\right\}\right)
$$

where $\mathbf{H}$ represents $\mathbf{H}_{\mathrm{sr}}^{(i)}, \mathbf{H}_{\mathrm{rd}}^{(i)} \forall i, \operatorname{SNR}_{m}^{(i)}(\mathbf{H})$ represents that it is a function of $\mathbf{H}$. Next, we focus on the behaviour of $f_{n_{i}}=\log _{2}\left(1+\mathrm{E}_{\mathbf{H}}\left\{\mathbf{S N R}_{m}^{(i)}(\mathbf{H})\right\}\right)$ as the number of relays $n_{i}$ of the $i$ th cluster increases. It can be assumed that for any set of values $\left\{v_{1}, v_{2}, v_{3} \in \mathbb{N} \mid v_{1}>v_{2}>v_{3}, v_{1}-v_{2}=v_{2}-v_{3}=1\right\}$, the following holds: $f_{v_{1}}-f_{v_{2}} \leq f_{v_{2}}-f_{v_{3}}$. This assumption is equivalent to

$$
\frac{1+\left.\mathrm{E}_{\mathbf{H}}\left\{\operatorname{SNR}_{m}^{(i)}(\mathbf{H})\right\}\right|_{n_{i}=v_{1}}}{1+\left.\mathrm{E}_{\mathbf{H}}\left\{\operatorname{SNR}_{m}^{(i)}(\mathbf{H})\right\}\right|_{n_{i}=v_{2}}} \leq \frac{1+\left.\mathrm{E}_{\mathbf{H}}\left\{\operatorname{SNR}_{m}^{(i)}(\mathbf{H})\right\}\right|_{n_{i}=v_{2}}}{1+\left.\mathrm{E}_{\mathbf{H}}\left\{\operatorname{SNR}_{m}^{(i)}(\mathbf{H})\right\}\right|_{n_{i}=v_{3}}}
$$

In [3], the authors showed that the capacity of a AF relay network can at most increase linearly with the number of relays, otherwise it is sublinear. Thus, we can deduce that in the most extreme case the linear behaviour of $f_{n_{i}}$ sufficiently means that (10) is satisfied with equality. As the rate flattens out with increasing $n_{i}$ [3], the inequality in (10) will be strictly smaller. In Fig. 3 the behaviour of $f_{n_{i}}$ and the strictness of the bound are validated numerically for several SNR values. After

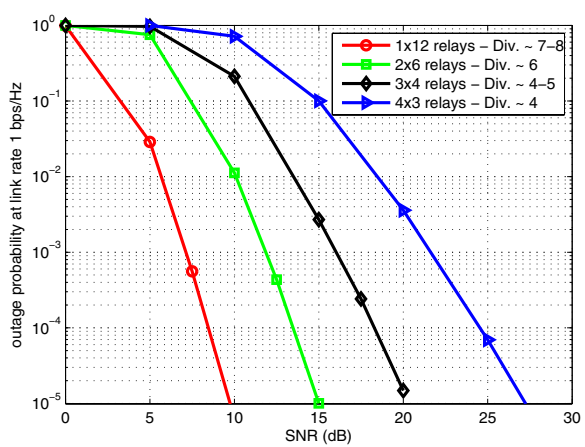

Fig. 4. The outage probability vs. SNR for different cluster configurations with $N_{r}=12, N=2$

validating our assumption, now we prove that OCC is optimum for maximizing the upper bound of average rate performance.

Assume that for given $C$ and $N_{r}$, there exists a cluster relay configuration $\tilde{\mathcal{C}}=\left\{m_{1}, \ldots, m_{C}\right\}$ with $\sum_{i=1}^{C} f_{m_{i}}$ which is larger than $\sum_{i=1}^{C} f_{n_{i}}$ obtained by the optimum cluster configuration $\mathcal{C}=\left\{n_{1}, \ldots, n_{C}\right\}$. Note that the term $1 /(2 C)$ is dropped. Let $m_{i}=n_{i}+r_{i}$ and $f_{m_{i}}=f_{n_{i}}+r_{i} \mu_{i}$. $\mu_{i}$ is defined as

$$
\mu_{i} \leq r_{i} \lambda_{1}, \quad r_{i} \geq 0 \quad \text { or } \quad \mu_{i} \leq r_{i} \lambda_{2}, \quad r_{i}<0,
$$

where $r_{i} \in \mathbb{Z}, \lambda_{1}=f_{n_{i}+1}-f_{n_{i}}, \lambda_{2}=f_{n_{i}}-f_{n_{i}-1}$, and $\lambda_{1} \leq \lambda_{2}$. The last condition for $\lambda_{1}, \lambda_{2}$ follows from the claim above. Next, following similar steps as for Proposition 1,

$$
\sum_{i=1}^{C} f_{m_{i}}=\sum_{i \in \mathcal{P}} r_{i} \lambda_{1}+\sum_{i \in \mathcal{P}^{\prime}} r_{i} \lambda_{2}+\sum_{i=1}^{C} f_{n_{i}}-\sum_{i=1}^{C} \varepsilon_{i},
$$

where $\varepsilon_{i} \in\left\{0, \mathbb{R}^{+}\right\} \forall i$. The first two summands of (11) are equal to

$$
\sum_{i \in \mathcal{P} \cap \mathcal{N}}\left|r_{i}\right| \lambda_{1}+\sum_{i \in \mathcal{P} \cap \mathcal{N}^{\prime}}\left|r_{i}\right| \tilde{\lambda}_{1}-\sum_{i \in \mathcal{P}^{\prime} \cap \mathcal{N}}\left|r_{i}\right| \lambda_{2}-\sum_{i \in \mathcal{P}^{\prime} \cap \mathcal{N}^{\prime}}\left|r_{i}\right| \tilde{\lambda}_{2}
$$

where $\tilde{\lambda}_{1}=f_{n_{i}}-f_{n_{i}-1}$ and $\tilde{\lambda}_{2}=f_{n_{i}-1}-f_{n_{i}-2}$. Since $\lambda_{1} \leq \tilde{\lambda}_{1}=\lambda_{2} \leq \tilde{\lambda}_{2}$, (12) can be written as

$$
\sum_{i \in \mathcal{P}} r_{i} \lambda_{1}+\sum_{i \in \mathcal{P}^{\prime}} r_{i} \lambda_{2}=\kappa\left(-\sum_{i \in \mathcal{P} \cap \mathcal{N}}\left|r_{i}\right|-\sum_{i \in \mathcal{P}^{\prime} \cap \mathcal{N}^{\prime}}\left|r_{i}\right|\right) \leq 0
$$

for $\kappa \in\left\{0, \mathbb{R}^{+}\right\}$. Combining (11) and (13), we have $\sum_{i=1}^{C} f_{m_{i}} \leq \sum_{i=1}^{C} f_{n_{i}}$, which contradicts with our assumption and concludes the proof.

\section{Simulation Results}

In this section, we present Monte-Carlo simulation results. The assumed CSI knowledge per node is as described in Section II. Furthermore, a local phase reference is assumed to be available inside the cluster. The average $\mathrm{SNR}=\sigma_{s}^{2} / \sigma_{n}^{2}=$ $20 \mathrm{~dB}$, and $\sigma_{s}^{2}=1$.

In Fig. 4, the outage probability versus SNR is plotted for different cluster configurations with $N_{r}=12, N=2$, and equally sized clusters. The case $C=1$ is equivalent to MU-ZF relaying with GCSI, which gives about 8th order diversity. As the network is divided into 2(3) relay clusters with 6(4) relays, the system is supposed to give at least $2 \mathrm{nd}(3 \mathrm{rd})$ order 

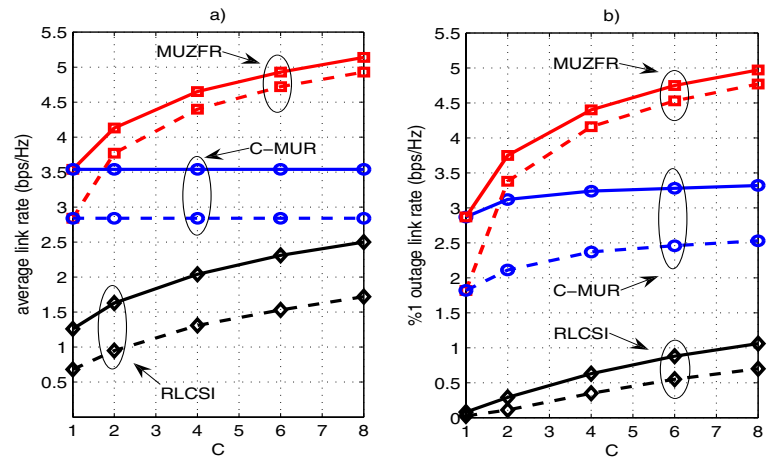

Fig. 5. a) Average rates vs. $\mathrm{C}$ b) Outage rates load vs. $\mathrm{C}$ ( $N=2$ (solid lines), $N=3$ (dashed lines), $n_{i}=9 \forall i$ ).
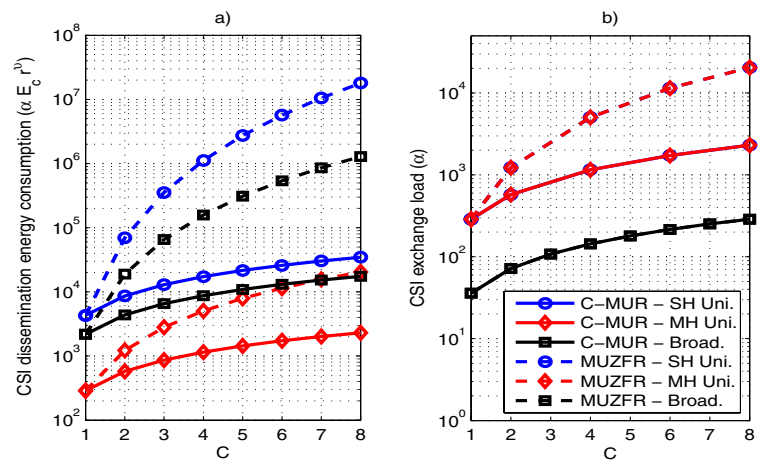

Fig. 6. a) Energy consumption of CSI dissemination vs. $C$ b) CSI exchange load vs. $C$ for unicast and broadcast schemes $\left(N=2, n_{i}=9 \forall i\right)$.

of diversity arrising from the phase rotations between clusters. The additional 4(2) orders of diversity come from the intra cluster gain allocations. This is consistent with the results of [5] presenting that maxmin rate gain allocation maximizes the diversity in MUZFR applications. It is noticable that for 4 clusters with 3 relays, the system offers only 4 th order of phase diversity, but no intra cluster diversity. It is also realized that clustering inevitabley suffers from array gain.

Fig. 5 presents a comparison between the proposed C-MUR, MUZFR [5] and relaying with only local CSI (RLCSI) at the relays [4]. Both C-MUR and MUZFR use maxmin rate gain allocations. Equally sized clusters each with 9 relays are considered. For any $C$, MUZFR and RLCSI use $9 C$ relays. Fig. 5-a shows the average link rates, and depicts that C-MUR acts as a balancing system between GCSI and LCSI systems. As new clusters are added to the system, the reliability of the C-MUR system increases. This improvement arises from the phase diversity. Also note that adding new clusters does not change the average link rates. It is seen from Fig. 5 that CMUR has the disadvantage of reduced rates w.r.t. MUZFR. But as expected, its advantage evinces itself in terms of drastically reduced LCSI overhead and transmit energy consumption for the dissemination. Fig. 6 depicts this trade-off. For $C=$ 8 , the C-MUR system offers 3,2,1 orders of magnitude energy consumption reduction for single-hop unicast, multihop unicast, and broadcast schemes, respectively. Besides, the CSI exchange overhead of C-MUR with the unicast scheme is only 10 percent of that of MUZFR for $C=8$. On the contrary, both systems have the same overhead with the broadcast
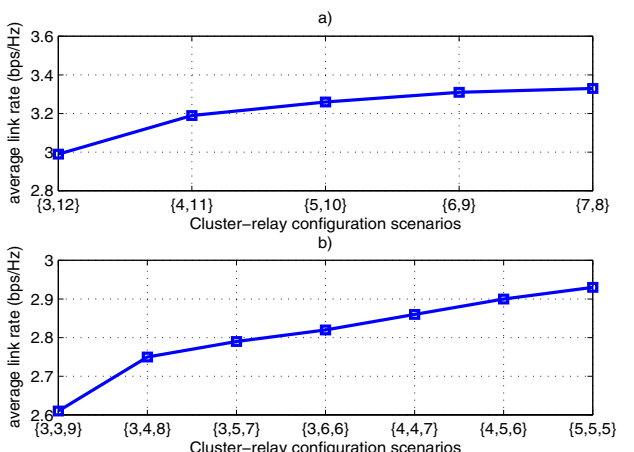

Fig. 7. Average rate vs. cluster-relay configuration: a) $C=2$, b) $C=3$ for $N_{s}=2, N_{r}=15$.

scheme. Establishing multiple hops to disseminate LCSI does not change the LCSI overhead w.r.t the single-hop unicast scheme. Note also that RLCSI has neither CSI exchange load nor energy consumption because the relays do not exchange LCSI.

Fig. 7 shows the numerical validation of the proof that the OCC maximizes the average link rates. The average link rate versus the cluster-relay configuration scenarios is plotted for $C=2$ and $C=3$. The x-axis consists of all possible cluster-relay configurations $\left\{n_{1}, \ldots, n_{C}\right\}$ for given $N_{r}$ and $C$. The maximum average link rates are achieved with the most uniform distribution of relays to clusters which are $\{7,8\}$, and $\{5,5,5\}$ for $C=2$ and $C=3$, respectively.

\section{CONCLUSION}

We proposed a novel cluster based MU relaying system with phase diversity. The relay gains were allocated per cluster such that the minimum link rate is maximized. A detailed analysis of CSI dissemination has been done. We concluded that for a given number of clusters and relays, the OCC mimimizes the CSIEL and dissemination energy consumption; and moreover maximizes the upper bound of average link rates. The cluster based system is a candidate to compansate the disadvantages of MUZFR with GCSI and RLCSI.

\section{REFERENCES}

[1] I. Hammerström, M. Kuhn, and A. Wittneben, "Cooperative diversity by relay phase rotations in block fading environments," in Proc. of IEEE Workshop on Sig. Proces. Adv. in Wir. Commun., July 2004, pp. 293-297.

[2] C. Akcaba, P. Kuppinger, and H. Bölcskei, "Distributed transmit diversity in relay networks," in Proc. of IEEE ITW, Vol. 3, July 2007.

[3] R. U. Nabar, Ö. Oyman, H. Bölcskei , and A. J. Paulraj, "Capacity scaling laws in MIMO wireless networks," in Proc. Allerton Conf. on Commun., Cont. and Comp., Oct. 2003, pp. 378-389.

[4] A. Wittneben and B. Rankov, "Distributed antenna systems and linear relaying for gigabit MIMO wireless," in Proc. IEEE Veh. Tech. Conf. Fall, Los Angeles, Sep. 2004.

[5] C.Eşli, Stefan Berger, and A. Wittneben, "Optimizing zero-forcing based gain allocation for wireless multiuser networks," in Proc. IEEE Inter. Conf. on Commun., Glasgow, July 2007.

[6] X. Zhong, R. Khosla, G. Khanna, S. Bagchi and E. J. Coyle, "DataCentric Routing in Sensor Networks: Single-hop Broadcast or Multi-hop Unicast?," in Proc. of IEEE 65th Veh. Tech. Conf., Spring 2007.

[7] J. F. Sturm, "Using SeDuMi 1.02, a Matlab toolbox for optimization over symmetric cones," Opt. Meth. and Soft., Vol. 11-12, 1999, pp. 625-653.

[8] N. D. Sidiropolous, T. N. Davidson and Z. H. T. Luo, "Transmit beamforming for physical-layer multicasting", IEEE Trans. on Sig. Proc., vol. 54 , no. 6 , pp. 2239-2251, June 2006 